\title{
Perfil epidemiológico da mortalidade masculina no Brasil, 2014-2018
}

\author{
Epidemiological profile of male mortality in Brazil, 2014-2018 \\ Perfil epidemiológico de la mortalidad masculina en Brasil, 2014-2018
}

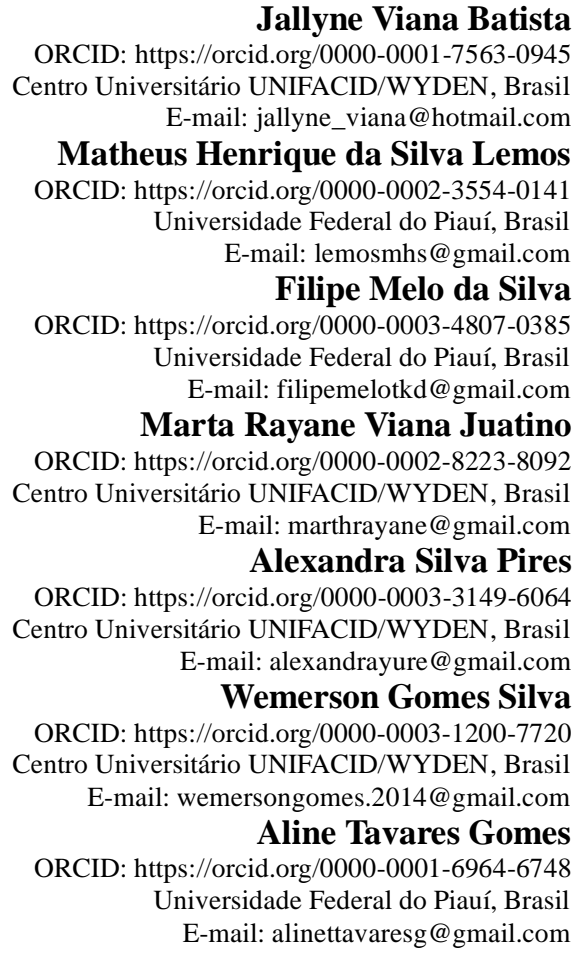

\begin{abstract}
Resumo
A política nacional de atenção à saúde do homem visa integrar à população masculina aos serviços de saúde, pois esse público-alvo não procura com muita frequência atendimento médico em razão dos aspectos culturais, vergonha ou falta de tempo. Assim, temos como problemática a alta mortalidade do sexo masculino. O presente estudo tem como objetivo geral, analisar o perfil epidemiológico da mortalidade masculina, e, como objetivo, especificar e identificar as causas da mortalidade entre os homens, além de descrever o perfil sócio demográfico à faixa etária, cor/raça, escolaridade e as macrorregiões. Esse trabalho se trata de um estudo descritivo, retrospectivo, de abordagem quantitativa. A pesquisa foi realizada com dados disponibilizados no sistema DATASUS no qual foram analisadas as principais causas de óbitos na população masculina entre 2014 a 2018, com fins de identificar e analisar as maiores causas epidemiológicas de óbitos em homens. Os resultados do estudo evidenciam que entre 2014 a 2018 teve 3.607.966 mil óbitos em homens. E que a região sudeste teve o maior percentual de óbitos entre as macrorregiões com $44,11 \%$. Quanto à faixa etária 80 anos ou mais com 21,76\%. Com relação cor/raça predominou a branca com 47,67\%. Escolaridade que predomina ficou de 1 a 3 anos 23,08\%, ignorados com 22,98\%. A pesquisa poderá reforçar os dados já obtidos por outros pesquisadores, ou, ainda, demonstrar outros índices ainda não levantados. Conclui-se a maior frequência de mortes masculinas por causas evitáveis, destaca-se a importância de investimentos em promoção da saúde e prevenção de agravos na atenção primária.
\end{abstract}

Palavras-chave: Perfil epidemiológico; Mortalidade; Masculino.

\begin{abstract}
The national policy of attention to men's health aims to integrate the male population to health services, as this target audience does not often seek medical attention due to cultural aspects, shame or lack of time. Thus, we have as a problem the high male mortality. The present study has the general objective of analyzing the epidemiological profile of male mortality, and, as an objective, to specify and identify the causes of mortality among men, in addition to describing the socio-demographic profile of the age group, color / race, education and macroregions. This work is a descriptive, retrospective study, with a quantitative approach. The research was carried out with data made available in the DATASUS system in which the main causes of death in the male population between 2014 and 2018 were analyzed, in order to identify and analyze the major epidemiological causes of deaths in men. The results of the study
\end{abstract}


show that between 2014 and 2018 there were 3,607,966 deaths in men. And that the southeast region had the highest percentage of deaths among the macro-regions with $44.11 \%$. As for the age group 80 years or more with $21.76 \%$. Regarding color / race, white predominated with $47.67 \%$. The predominant level of education was 1 to 3 years $23.08 \%$, ignored with $22.98 \%$. The research may reinforce the data already obtained by other researchers, or, still, demonstrate other indices not yet surveyed. It concludes the greater frequency of male deaths from preventable causes, the importance of investments in health promotion and disease prevention in primary care is highlighted.

Keywords: Health profile; Mortality; Male.

\section{Resumen}

La política nacional de atención a la salud masculina tiene como objetivo la integración de la población masculina a los servicios de salud, ya que este público objetivo no suele buscar atención médica por aspectos culturales, vergüenza o falta de tiempo. Por tanto, tenemos como problema la alta mortalidad masculina. El presente estudio tiene el objetivo general de analizar el perfil epidemiológico de la mortalidad masculina, y, como objetivo, especificar e identificar las causas de mortalidad entre los hombres, además de describir el perfil sociodemográfico del grupo de edad, color / raza, educación y macrorregiones. Este trabajo es un estudio descriptivo, retrospectivo, con enfoque cuantitativo. La investigación se realizó con datos disponibles en el sistema DATASUS en el que se analizaron las principales causas de muerte en la población masculina entre 2014 y 2018, con el fin de identificar y analizar las principales causas epidemiológicas de muerte en hombres. Los resultados del estudio muestran que entre 2014 y 2018 hubo 3.607.966 muertes en hombres. Y que la región sureste tuvo el mayor porcentaje de muertes entre las macrorregiones con 44,11\%. En cuanto al grupo de edad de 80 años o más con un $21,76 \%$. En cuanto al color / raza, predominó el blanco con un 47,67\%. El nivel educativo predominante fue de 1 a 3 años 23,08\%, ignorado con 22,98\%. La investigación puede reforzar los datos ya obtenidos por otros investigadores o, aún así, demostrar otros índices aún no encuestados. Se concluye la mayor frecuencia de muertes masculinas por causas prevenibles, se destaca la importancia de las inversiones en promoción de la salud y prevención de enfermedades en la atención primaria.

Palabras clave: Perfil de salud; Mortalidad; Masculino.

\section{Introdução}

A Política Nacional de Atenção Integral à Saúde do Homem PNAISH, instituída pela Portaria GM/MS n 1944, de 27 de agosto de 2009, veio para inserir essa parcela da população na atenção primária, assim, reduzir o índice de morbimortalidade masculina, considerado alto em relação ao feminino, que representar um grande problema na saúde pública, tento em vista a resistência a procura ao atendimento por aspectos culturais de que o homem não pode adoecer porque fere sua masculinidade, com isso ignora a importância a prevenção de doenças (Cavalcante, et al., 2014).

A PNAISH tem como objetivo a promoção de ações de saúde que possibilitem a apreensão da realidade dos homens entre 20 e 59 anos de idade nos seus diversos contextos. Facilitar e amplia o acesso da população masculina aos serviços. A Política Nacional de Atenção Integral à Saúde do Homem é desenvolvida a partir de cinco eixos temáticos, são eles: acesso e acolhimento, saúde sexual e saúde reprodutiva, paternidade e cuidado, doenças prevalentes na população masculina, prevenção de violências e acidentes. Apesar da política já ter 10anos que foi criada ainda não foi visto mudanças no comportamento da população masculina em procurar atendimento médico (Brasil, 2009).

O homem sempre foi visto como o provedor da família, um dos princípios destacados é a obrigação de mudança do pensamento masculina nos cuidados com a própria saúde, isso se deve muito ao machismo pelo pensamento de que o homem não pode adoecer e tem que cuidar da família, por pensamentos assim que o homem tem a menor expectativa de vida e se coloca vulnerável frente a uma série de doenças, que poderia ser evitada com atividades educativas se prevenindo ao procurar atendimento médico (Separavich, et al., 2013).

A maioria dos homens entra no sistema de saúde por meio da atenção especializada, os mesmo com o pior índice populacional de morbimortalidade com doenças crônicas gerando um gasto muito alto na rede hospitalar, tendo como consequência o agravo da morbidade e o aumento da mortalidade, é necessário fortalecer, informar e qualificar a atenção primária garantindo, assim, a promoção e prevenção da saúde e dos problemas evitáveis (Silveira, Melo \& Barreto, 2017). 
Nos lugares onde homens e mulheres enfrentam a mesma doença, eles têm expectativa menor. Entres as principais causas de morte, 33 contribuem mais para reduzir a expectativa de vida em homens do que em mulheres. Em 2016, a perspectiva de uma pessoa de 30 anos morrerem de uma doença não transmissível antes dos 70 anos de idade foi $44 \%$ maior em homens do que em mulheres (Organização pan-americana de saúde, 2019).

As doenças que mais acometem os homens, segundo o DATASUS, são doenças do aparelho circulatório (DAC), neoplasias malignas (NM), doenças do aparelho respiratório (DAR) e as causas externas (CE), doenças essas, que poderiam ser evitadas na grande maioria dos casos ao ser descoberta no início, se o homem cuidasse mais do seu corpo e procurasse atendimento médico primário com maior frequência (Batista et al., 2017).

O presente estudo tem como objetivo analisar o perfil epidemiológico da mortalidade masculina no Brasil afim de conhecer e analisar as doenças que o sexo masculino está mais acometido, a saúde do homem deve ser abordada como um assunto de grande importância já que os índices de mortalidade são bem elevados em consideração às mulheres.

\section{Metodologia}

Trata-se de um estudo epidemiológico e descritivo com abordagem quantitativa, foi realizado nos anos de 2014-2018, A partir do banco de dados do sistema de informação sobre mortalidade (SIM) - banco de dados de domínio público DATASUS, formado por dados da declaração de óbitos, disponível pela internet sem identificação dos indivíduos. Para Pereira, et al., (2018) no método quantitativo, a coleta de dados é realizada a partir de dados quantitativos ou numéricos e são analisados por meio de técnicas matemáticas como porcentagens, estatísticas e probabilidades.

A pesquisa teve como cenário de estudo o banco de dados de domínio público (DATASUS) e por tratar-se de um banco de dados de domínio público, disponibilizado pelo SUS, não houve necessidade de submissão do trabalho ao Comitê de Ética e Pesquisa (CEP), estabelecidos na Resolução 466/12 do Conselho Nacional de Saúde.

A população do referido estudo foi composta por todos os óbitos em homens no Brasil, (CID-10). Para a seleção dos sujeitos da pesquisa foram adotados alguns critérios de inclusão: todos os óbitos em homens e suas principais causas de mortalidade entre 2014 a 2018 e os dados sócio demográfico.

A coleta de dados foi realizada de novembro a dezembro de 2019, a partir do banco de dados do sistema de informação sobre mortalidade (SIM) - DATASUS, formado por dados das declarações de óbitos, disponibilizado pela internet entre os anos de 2014-2018.

As variáveis estudadas foram: sexo masculino, idade, local de ocorrência do óbito entre as macrorregiões do Brasil (Sudeste, Sul, Centro-Oeste, Nordeste e Norte), cor/raça (preta, amarela, branca, parda, indígena e ignorado), escolaridade (nenhuma, 1 a 3 anos, 4 a 7 anos, 8 a 11 anos, 12 anos ou mais e ignorados), e as principais causas de óbitos em homens que são causas externas classificadas de acordo com o Capítulo XX, da 10ª Classificação Internacional de Doenças - CID-10, as Neoplasias classificadas de acordo com o capitulo CID-10 (II), Doença do aparelho circulatório classificadas de acordo com o capitulo CID-10 (IX), doença do aparelho respiratório classificadas de acordo com o capitulo CID-10 (X).

Os dados foram tabulados diretamente em planilha eletrônica desenvolvida no programa Microsoft Excel 2010, construída especialmente para a pesquisa. Para melhor visualização dos resultados, foram construídos gráficos e tabelas. Tais dados foram confrontados com a literatura científica existente sobre a temática.

\section{Resultados}

Nota-se que no período de 2014 a 2018, houve 3.607.966 óbitos no sexo masculino. Destes, a região Sudeste apresentou o maior percentual de óbitos (44,11\%), seguida da região Nordeste (26,97\%). Podemos relatar ainda que entre 2017 a 2018 as regiões Nordeste e centro-oeste tiveram uma tendência descrente. (Quadro 1). 
Quadro 1 - Distribuição da frequência de óbitos no sexo masculino, nas macrorregiões do Brasil, 2014-2018.

\begin{tabular}{|c|c|c|c|c|c|c|c|c|c|c|c|c|}
\hline & \multicolumn{2}{|c|}{2014} & \multicolumn{2}{|c|}{2015} & \multicolumn{2}{|c|}{2016} & \multicolumn{2}{|c|}{2017} & \multicolumn{2}{|c|}{2018} & \multicolumn{2}{|c|}{ TOTAL } \\
\hline & $\mathbf{N}$ & $\%$ & $\mathbf{N}$ & $\%$ & $\mathbf{N}$ & $\%$ & $\mathbf{N}$ & $\%$ & $\mathbf{N}$ & $\%$ & $\mathbf{N}$ & $\%$ \\
\hline Norte & 45.992 & 6,63 & 47.950 & 6,76 & 49.725 & 6,75 & 50.937 & 6,94 & 51.568 & 7,03 & 246.172 & 6,82 \\
\hline Nordeste & 185.065 & 26,67 & 192.385 & 27,13 & 199.015 & 27,01 & 201.255 & 27,40 & 195.296 & 26,62 & 973.016 & 26,97 \\
\hline Sudeste & 308.586 & 44,47 & 312.711 & 44,10 & 324.899 & 44,09 & 321.540 & 43,78 & 323.636 & 44,12 & 1.591 .372 & 44,11 \\
\hline Sul & 105.188 & 15,16 & 106.378 & 15,00 & 112.466 & 15,26 & 109.740 & 14,94 & 112.244 & 15,30 & 546.016 & 15,13 \\
\hline Centro-Oeste & 49.091 & 7,07 & 49.693 & 7,01 & 50.737 & 6,89 & 50.997 & 6,94 & 50.872 & 6,93 & 251.390 & 6,97 \\
\hline Total & 693.922 & 100 & 709.117 & 100 & 736.842 & 100 & 734.469 & 100 & 733.616 & 100 & 3.607 .966 & 100 \\
\hline
\end{tabular}

Fonte: DATASUS/SIM.

Verificou-se que o maior percentual de óbitos se encontra na faixa etária de 80 e+ (21,76\%). Diferente da faixa etária de 5 a 9 anos que se encontra com o menor percentual de óbitos (0,26\%). Observa-se ainda que nas faixas etária de menor de 1 ano, 10 a 14 anos, 15 a 19 anos, 30 a 39 anos, 40 a 49 anos e ignorados tiveram uma redução no número de óbitos em todos os anos do estudo. (Quadro 2). 
Quadro 2 - Distribuição de óbito no sexo masculino, de acordo com a faixa etária no Brasil, 2014-2018.

\begin{tabular}{|c|c|c|c|c|c|c|}
\hline Faixa Etária & 2014 & 2015 & 2016 & 2017 & 2018 & TOTAL \\
\hline Menor de 1 ano & $\begin{array}{c}21.366 \\
(3,08 \%)\end{array}$ & $\begin{array}{l}20.683 \\
(2,92 \%)\end{array}$ & $\begin{array}{l}20.191 \\
(2,74 \%)\end{array}$ & $\begin{array}{l}19.846 \\
(2,70 \%)\end{array}$ & $\begin{array}{l}19.629 \\
(2,68 \%)\end{array}$ & $\begin{array}{l}101.715 \\
(2,82 \%)\end{array}$ \\
\hline 1 a 4 anos & $\begin{array}{c}3.308 \\
(0,48 \%)\end{array}$ & $\begin{array}{c}3.024 \\
(0,43 \%)\end{array}$ & $\begin{array}{c}3.362 \\
(0,46 \%)\end{array}$ & $\begin{array}{c}3.223 \\
(0,44 \%)\end{array}$ & $\begin{array}{c}3.149 \\
(0,43 \%)\end{array}$ & $\begin{array}{l}16.066 \\
(0,45 \%)\end{array}$ \\
\hline 5 a 9 anos & $\begin{array}{c}2.098 \\
(0,30 \%)\end{array}$ & $\begin{array}{c}1.821 \\
(0,26 \%)\end{array}$ & $\begin{array}{c}1.859 \\
(0,25 \%)\end{array}$ & $\begin{array}{c}1.893 \\
(0,26 \%)\end{array}$ & $\begin{array}{c}1.745 \\
(0,24 \%)\end{array}$ & $\begin{array}{c}9.416 \\
(0,26 \%)\end{array}$ \\
\hline 10 a 14 anos & $\begin{array}{c}3.351 \\
(0,48 \%)\end{array}$ & $\begin{array}{c}3.046 \\
(0,43 \%)\end{array}$ & $\begin{array}{c}3.009 \\
(0,41 \%)\end{array}$ & $\begin{array}{c}2.828 \\
(0,39 \%)\end{array}$ & $\begin{array}{c}2.596 \\
(0,35 \%)\end{array}$ & $\begin{array}{l}14.830 \\
(0,41 \%)\end{array}$ \\
\hline 15 a 19 anos & $\begin{array}{c}18.192 \\
(2,62 \%)\end{array}$ & $\begin{array}{c}17.480 \\
(2,47 \%)\end{array}$ & $\begin{array}{c}17.988 \\
(2,44 \%)\end{array}$ & $\begin{array}{c}17.763 \\
(2,42 \%)\end{array}$ & $\begin{array}{l}15.604 \\
(2,13 \%)\end{array}$ & $\begin{array}{l}87.027 \\
(2,41 \%)\end{array}$ \\
\hline 20 a 29 anos & $\begin{array}{c}45.628 \\
(6,58 \%)\end{array}$ & $\begin{array}{c}44.144 \\
(6,23 \%)\end{array}$ & $\begin{array}{c}45.248 \\
(6,14 \%)\end{array}$ & $\begin{array}{l}45.870 \\
(6,25 \%)\end{array}$ & $\begin{array}{l}42.260 \\
(5,76 \%)\end{array}$ & $\begin{array}{l}223.150 \\
(6,18 \%)\end{array}$ \\
\hline 30 a 39 anos & $\begin{array}{c}46.881 \\
(6,76 \%)\end{array}$ & $\begin{array}{l}45.998 \\
(6,49 \%)\end{array}$ & $\begin{array}{l}46.230 \\
(6,27 \%)\end{array}$ & $\begin{array}{l}45.822 \\
(6,24 \%)\end{array}$ & $\begin{array}{l}43.582 \\
(5,94 \%)\end{array}$ & $\begin{array}{l}228.513 \\
(6,33 \%)\end{array}$ \\
\hline 40 a49 anos & $\begin{array}{c}60.053 \\
(8,65 \%)\end{array}$ & $\begin{array}{c}59.739 \\
(8,42 \%)\end{array}$ & $\begin{array}{c}60.178 \\
(8,17 \%)\end{array}$ & $\begin{array}{c}58.165 \\
(7,92 \%)\end{array}$ & $\begin{array}{l}57.632 \\
(7,86 \%)\end{array}$ & $\begin{array}{l}295.767 \\
(8,20 \%)\end{array}$ \\
\hline 50 a 59 anos & $\begin{array}{c}94.131 \\
(13,57 \%)\end{array}$ & $\begin{array}{c}95.354 \\
(13,45 \%)\end{array}$ & $\begin{array}{c}99.031 \\
(13,44 \%)\end{array}$ & $\begin{array}{c}95.703 \\
(13,03 \%)\end{array}$ & $\begin{array}{c}96.725 \\
(13,18 \%)\end{array}$ & $\begin{array}{c}480.944 \\
(13,33 \%)\end{array}$ \\
\hline 60 a 69 anos & $\begin{array}{c}119.279 \\
(17,19 \%)\end{array}$ & $\begin{array}{c}124.492 \\
(17,56 \%)\end{array}$ & $\begin{array}{c}132.642 \\
(18,00 \%)\end{array}$ & $\begin{array}{c}133.125 \\
(18,13 \%)\end{array}$ & $\begin{array}{c}136.507 \\
(18,61 \%)\end{array}$ & $\begin{array}{c}646.045 \\
(17,91 \%)\end{array}$ \\
\hline 70 a 79 anos & $\begin{array}{c}133.110 \\
(19,18 \%)\end{array}$ & $\begin{array}{c}137.813 \\
(19,43 \%)\end{array}$ & $\begin{array}{c}143.836 \\
(19,52 \%)\end{array}$ & $\begin{array}{c}144.218 \\
(19,64 \%)\end{array}$ & $\begin{array}{c}147.693 \\
(20,13 \%)\end{array}$ & $\begin{array}{c}706.670 \\
(19,59 \%)\end{array}$ \\
\hline 80 e+ & $\begin{array}{c}143.646 \\
(20,70 \%)\end{array}$ & $\begin{array}{c}152.923 \\
(21,57 \%)\end{array}$ & $\begin{array}{c}160.703 \\
(21,81 \%)\end{array}$ & $\begin{array}{c}163.660 \\
(22,28 \%)\end{array}$ & $\begin{array}{c}164.294 \\
(22,40 \%)\end{array}$ & $\begin{array}{c}785.226 \\
(21,76 \%)\end{array}$ \\
\hline Ignorados & $\begin{array}{c}2.879 \\
(0,41 \%)\end{array}$ & $\begin{array}{c}2.600 \\
(0,37 \%)\end{array}$ & $\begin{array}{c}2.565 \\
(0,35 \%)\end{array}$ & $\begin{array}{c}2.353 \\
(0,32 \%)\end{array}$ & $\begin{array}{c}2.200 \\
(0,30 \%)\end{array}$ & $\begin{array}{c}12.597 \\
(0,35 \%)\end{array}$ \\
\hline Total & $\begin{array}{c}\mathbf{6 9 3 . 9 2 2} \\
100 \%\end{array}$ & $\begin{array}{c}\mathbf{7 0 9 . 1 1 7} \\
100 \%\end{array}$ & $\begin{array}{c}\mathbf{7 3 6 . 8 4 2} \\
100 \%\end{array}$ & $\begin{array}{c}\mathbf{7 3 4 . 4 6 9} \\
100 \%\end{array}$ & $\begin{array}{c}\mathbf{7 3 3 . 6 1 6} \\
100 \%\end{array}$ & $\begin{array}{c}\mathbf{3 . 6 0 7 . 9 6 6} \\
100 \%\end{array}$ \\
\hline
\end{tabular}

Fonte: DATASUS/SIM.

Com relação à cor/raça, nota-se que a branca apresentou um destaque maior com $(47,67 \%)$, seguida da parda $(8,84 \%)$ e ignorada com $(3,81 \%)$. (Gráfico 1$)$. 
Research, Society and Development, v. 10, n. 5, e51710515248, 2021

(CC BY 4.0) | ISSN 2525-3409 | DOI: http://dx.doi.org/10.33448/rsd-v10i5.15248

Gráfico 1 - Distribuição de óbito em homens segundo cor/raça no Brasil, 2014-2018.

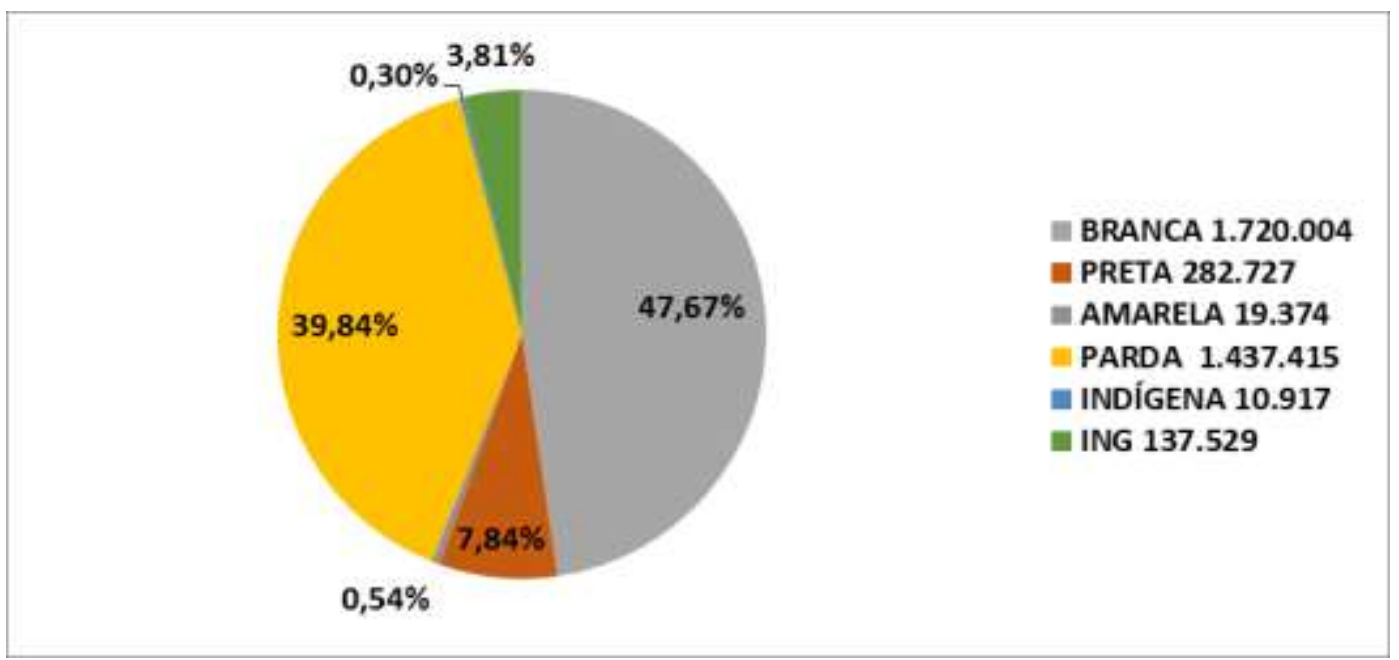

Fonte: DATASUS/SIM.

Observou-se que a escolaridade de 1 a 3 anos apresentou o maior percentual (23,08\%), seguida da escolaridade ignorada (22,98\%). Com relação ao menor percentual apresentado, temos a escolaridade de 12 anos e mais $(4,32 \%)$. (Gráfico 2).

Gráfico 2 - Distribuição de óbito no sexo masculino, segundo escolaridade, Brasil, 2014-2018.

\begin{tabular}{|c|c|c|c|c|c|c|}
\hline $25,00 \%$ & \multirow[b]{3}{*}{$13,33 \%$} & $23,08 \%$ & & \multirow[b]{3}{*}{$14,32 \%$} & & $22,98 \%$ \\
\hline $20,00 \%$ & & & & & & \\
\hline $15,00 \%$ & & & & & & \\
\hline $10,00 \%$ & & & & & & \\
\hline $5,00 \%$ & & & & & $4,83 \%$ & \\
\hline $0,00 \%$ & Nenhuma & 1 a 3 anos & 4 a 7 anos & 8 a 11 anos & 12 anos e mais & Ignorados \\
\hline
\end{tabular}

Fonte: DATASUS/SIM.

Observa-se que o maior percentual de óbitos se refere às doenças do aparelho circulatório com (36,84\%), seguida das causas externas $(25,20 \%)$. Nota-se ainda que nos últimos três anos estudados as neoplasias apresentaram uma tendência de crescimento. (Quadro 3). 
Quadro 3 - Distribuição de óbitos no sexo masculino, de acordo com as principais doenças relacionadas ao homem, Brasil, 2014-2018.

\begin{tabular}{|c|c|c|c|c|c|c|c|}
\hline & 2014 & 2015 & 2016 & 2017 & 2018 & To & \\
\hline & $\%$ & $\%$ & $\%$ & $\%$ & $\%$ & $\mathbf{N}$ & $\%$ \\
\hline $\begin{array}{l}\text { Causas } \\
\text { Externas }\end{array}$ & 26,56 & 25,23 & 24,93 & 25,31 & 24,04 & 634.365 & 25,20 \\
\hline Neoplasias & 22,06 & 22,45 & 22,22 & 22,72 & 23,41 & 568.338 & 22,58 \\
\hline $\begin{array}{l}\text { Doenças do } \\
\text { aparelho } \\
\text { Respiratório }\end{array}$ & 14,72 & 15,42 & 15,84 & 15,35 & 15,53 & 387.065 & 15,38 \\
\hline $\begin{array}{l}\text { Doenças do } \\
\text { aparelho } \\
\text { Circulatório }\end{array}$ & 36,66 & 36,90 & 37,01 & 36,62 & 37,02 & 927.354 & 36,84 \\
\hline Total & $\begin{array}{c}100 \\
(n=486.342)\end{array}$ & $\begin{array}{c}100 \\
(n=494.778)\end{array}$ & $\begin{array}{c}100 \\
(n=514.091)\end{array}$ & $\begin{array}{c}100 \\
(n=513.253)\end{array}$ & $\begin{array}{c}100 \\
(n=508.658)\end{array}$ & 2.517.122 & 100 \\
\hline
\end{tabular}

Fonte: DATASUS/SIM.

\section{Discussão}

A saúde do homem vem ganhado importância no cenário mundial, sendo de extrema importância a discussão sobre a temática. A mesma merece atenção a parti do momento que surgem diferenças nas evidências sobre as tendências epidemiológicas nos gêneros masculinos e femininos (Organização Pan-americana de Saúde, 2018).

Apesar dos altos índices de mortalidade, os homens continuam não procurando o serviço preventivo, no qual é mais procurado pelas mulheres, e continuam adentrando os serviços de alta e média complexidade (Carneiro, Adjunto \& Alves, 2019). Esta atitude tem corroborado para o elevado número de óbitos, como se é observado em todas as macrorregiões do Brasil.

Segundo Oliveira et al., (2017), entre 1980 e 2009 houve uma diminuição de 32\% dos óbitos na faixa etária mais jovem, ao passo que, na faixa etária entre 40 a 59 anos houve um aumento de 13\%. Tal achado vai de encontro com os dados apresentados no presente estudo que mostra um predomínio maior na faixa etária 80 e mais. Oliveira et al., ainda enfatiza que as diferenças de mortalidade em relação a faixa etária, podem estar relacionadas a similaridade de comportamento, particularmente no que trata de hábitos de risco, como exposição ao tabagismo e estresse ocupacional.

A raça/cor no Brasil é uma informação que passou a ser contada para medir a situação social dos indivíduos, e os riscos associados à sua morbimortalidade. Assim nos anos entre 2002 a 2010, no Brasil, ocorreu um aumento de óbitos referente a homicídio nas pessoas que se declarou na cor/raça preta $5,6 \%$ e uma diminuição de $24,8 \%$ na cor/raça branco (Oliveira et al., 2017). Da mesma forma ocorreu nos dados do presente estudo, onde a maioria dos óbitos eram de cor/raça branca.

Em relação à escolaridade, este estudo mostrou que existe um percentual maior relacionado a 1 a 3 anos de estudos. Desta forma um estudo realizado por Silva, Freire e Pereira (2010), mostrou que as chances de óbitos são menores em homens com maiores níveis de escolaridades, sendo explicado pela desigualdade econômica, diferença de acesso em saúde, estilo de vida e hábitos alimentares.

De acordo com Pereira et al., (2015), a escolaridade é um fator importante na aplicação de programas e ações efetivas para promoção da saúde do homem, uma vez que quanto menor escolaridade maior a dificuldade de compreensão em relação a fatores de riscos e preventivos a saúde do homem. 
As causas externas responderam em 2011 por aproximadamente 3,5 milhões de mortes entre indivíduos de 15 e 69 anos de idade, o que corresponde a 15,4\% da mortalidade global. No Brasil acomete cerca 28,6\% de óbitos por causas externas em homens no ano de 2010. Em relação à cor/raça, pessoas pardas tem maior probabilidade de mortes por causas externas que pessoas brancas. O estudo também fala que a população cor/raça pretos e pardos tem maiores risco de mortes por homicídio, do que pessoas brancas independente da escolaridade (Moura et al., 2014).

Por conta da frequência com que ocorrem e por serem os adolescentes e adultos jovens os grupos que mais se arriscam, as causas externas são os maiores responsáveis sobre os altos índices de mortalidade na população masculina, assim. Foi observado a predominância de mortes por acidente de transporte terrestre, suicídios e homicídios (Gonsaga et al., 2012).

Segundo Fraga et al., (2016), os óbitos por causas externas têm como principal faixa etário o grupo de adultos jovens entre 30 a 39 anos, tendo as causas violentas e acidentes de transporte como principais fatores que comentes os homens, e as doenças do aparelho circulatório e neoplasias malignas tendo a idade como cofator contribuinte para o aparecimento das mesmas, com isso quanto maior a idade maior a probabilidade de morrer por doenças do aparelho circulatório, respiratório e neoplasias.

Segundo Melo et al., (2019), o maior percentual de óbitos em indivíduos acima dos 60 anos está relacionado com o aumento da expectativa de vida da população atual, tendo maior morbidade e mortalidade na velhice. Tendo como principal causa de óbito doenças do aparelho circulatório e respiratório na velhice. Calderin (2016) corrobora com tal afirmação, onde foi observado que na cidade de São Paulo e outras cidades que devido a poluição ambiental e o tabagismo acarretaram no aumento do número de óbitos por doenças pulmonar obstrutiva crônica.

O padrão etário da mortalidade mostra que a distribuição dessas principais causas de mortes está concentrada, principalmente, nas idades avançadas. Tal situação já é esperada, uma vez que, com tendência de aumento de doenças degenerativas e causadas pelos homens, as faixas etárias que ficam mais vulneráveis são aqueles referentes aos idosos (Souza \& Siviero, 2015).

Assim determinadas condições masculinas, estão diretamente relacionadas a situações de vulnerabilidade ao adoecimento, principalmente a agravos crônicos. Tendo isso ido d encontro com um estudo realizado nos Estados Unidos que comparam a situação de saúde entre o sexo masculino e feminino, mostrando que os homens são mais susceptíveis ao adoecimento (Carneiro, Adjunto \& Alves, 2019).

Estudos enfatizam que os homens negligenciam a própria saúde mais que as mulheres, buscando alternativas de tratamento quando percebem alguma anormalidade. A citar a grande demanda dos mesmos nas farmácias imbuídos em buscar o tratamento rápido e que muitas vezes satisfaz os objetivos, que eles temiam como principais, ou de dar alívio (Schraiber, 2010 \& Figueiredo, 2013).

Desta forma devem-se haver cada vez mais investimentos na política de saúde do homem, a fim de mudar a visão de que os serviços de saúde têm como único propósito tratar as doenças. Deve-se existir também uma educação continuada e mais qualificada dos profissionais, além da realização de campanhas preventivas e promoção da saúde.

\section{Conclusão}

O presente estudo permitiu conhecer o perfil epidemiológico dos óbitos em homens entre as macrorregiões do Brasil. Assim observou-se que a região Sudeste foi a que ocorreu maior número de óbitos, bem como a faixa etária de 80 anos ou mais, homens com 1 a 3 anos de escolaridade e de cor/raça branca.

É importante ressaltar a importância dos registros das informações sobre óbitos, bem como da melhoria na coleta de dados e sistematização destes, visto que possibilitam uma melhor execução de políticas públicas brasileiras para prevenção e cuidados em saúde, promovendo a dignidade de toda a população. 
Desta forma os resultados obtidos nesta pesquisa nos permitiram concluir que o sexo masculino adoece com maior frequência, pois tem resistência na procura do serviço de saúde, sendo necessária a implantação de medidas essências voltadas para a educação e promoção da saúde a este público, podendo assim contribuir para a modificação do atual panorama de saúde do homem.

Espera-se que este estudo contribua no sentido de reforçar a importância de investigação e discussão da mortalidade masculina. Desta forma dada à importância do assunto, se faz necessário o desenvolvimento de novos estudos epidemiológico para que se tenha uma análise mais profunda sobre a mortalidade no homem e seus fatores causais.

\section{Referências}

Batista, J., Barreto, M. S., Merino, M. F.G. L., Fracasso, N. V., \& Baldissera, V. N. A. (2017). Perfil epidemiológico da Mortalidade por causas externas entre beneficiários de planos de saúde no brasil. Revista de enfermagem do centro oeste Mineiro. 8. e1870.

Brasil, Ministério da Saúde. (2009). Política nacional de atenção integral à saúde do homem. https://bvsms.saude.gov.br/bvs/publicacoes/politica_ nacional_atencao_integral_saude_homem.pdf

Calderin, J. C. I. (2016). Ação para redução da alta incidência de pacientes com doenças respiratórias. Trabalho de conclusão de curso (Especialização em Atenção Básica em Saúde da Família) - Universidade Federal de Minas Gerais. Juiz de Fora: MG. https://www.nescon.medicina.ufmg.br/biblioteca/imagem/a\%C3\%A7\%C3\%A3o-para_redu\%C3\%A7ao_da_alta_incidencia_de_pacientes.pdf

Carneiro, V. S. M., Adjuto, R. N. P., \& Alves, K.A.P. (2019). Saúde do homem: identificação e análise dos fatores relacionados à procura, ou não, dos serviços de atenção primária. Arquivos de Ciências da Saúde da UNIPAR. 23(1).

Cavalcanti, J. C. D., Ferreira, J. A., Henriques, A. H. B., Morais, G. S. N., Trigueiro, J. V. S., \& Torquato, I. M. B. (2014). Assistência Integral a Saúde do Homem: necessidades, obstáculos e estratégias de enfrentamento. Esc. Anna Nery, 18(4). 628-634.

Figueiredo, W. (2005) Assistência à saúde dos homens: um desafio para os serviços de atenção primária. Ciênc saúde coletiva. 10(1). 105-109.

Fraga, J. C. A. X. O. C, Áurea, C. P., Rocha, R. M, Silva, L. A, Medeiros, R. M. K., \& Mozer, I. T. (2016). Principais causas da mortalidade masculina e os anos potenciais de vida perdidos por estes agravos. Ciência, Cuidado \& Saúde, 15(4), 746-754.

Gonsaga, R. A. T., Rimoli, C. F., Pires, E. A., Zogheib, F. S., Fujino, M. V. T., \& Cunha, M. B. (2012). Avaliação da mortalidade por causas externas. Revista do Colégio Brasileiro de Cirurgiões, 39(4), 263-267.

Melo, C. S., Machado, D. P., Santos, I. K. P., Dias, C. A. G. M, Oliveira, E., Dendasck, C. V., \& Fecury, A. A. (2019). Caracterização epidemiológica dos óbitos no Brasil por macrorregião de 2016 a 2018. Revista Científica Multidisciplinar Núcleo do Conhecimento. 12(1). 05-17.

Moura, E. C., Gomes, R., Falcão, M. T. C., Schwarz, E., Neves, A. C. M., \& Santos, W. (2010). Desigualdades de gênero na mortalidade por causas externas no Brasil. Ciência \& Saúde Coletiva, 20(3). 779-788.

Oliveira, J., Corrêa, Á., Arruda e Silva, L., Mozer, I., \& Medeiros, R (2017). Perfil Epidemiológico da Mortalidade Masculina: Contribuições Para Enfermagem. Cogitare Enfermagem. 22(2).

Organização Pan-Americana da Saúde. (2018). Editorial: a importância de abordar a masculinidade e a saúde dos homens para avançar rumo à saúde universal e à igualdade de gênero. https:/www.paho.org/bra/index.php?option=com_content\&view=article\&id=5876:editorial-a-importancia-de-abordar-amasculinidade-e-a-saude-dos-homens-para-avancar-rumo-a-saude-universal-e-a-igualdade-de-genero $\&$ Itemid= $=820$

Organização Pan-americana da saúde. (2017). Ministério da saúde. Doenças cardiovasculares. https://www.paho.org/salud-en-las-americas-2017/wpcontent/uploads/2017/09/SA-2017-pt.pdf

Pereira, V. C. L. S., Pereira, M. M. M., Cézar, E. S. R., Braga, L. S., Espínola, L. L., \& Azevedo, E. B. (2015). Saúde do homem na atenção básica: análise acerca do perfil e agravos à saúde. Revista de Enfermagem UFPE on line. 9(1). 440-447

Pereira, A. S., Shitsuka, D. M., Parreira, F. J. \& Shitsuka, R. (2018). Metodologia da pesquisa científica. UFSM. https://repositorio.ufsm.br/bitstream /handle/1/15824/Lic_Computacao_Metodologia-Pesquisa-Cientifica.pdf?sequence $=1$..

Schraiber, L. B, Figueiredo, W. S, Gomes R, Couto, M. T, Pinheiro, T. F., \& Machin, R. (2015). Necessidades de saúde e masculinidades: atenção primária não cuidando de homens. Cad saúde pública. 26 (5). 961-70.

Separavich, M. A., \& Canesqui, A. M. (2013). Saúde do homem e masculinidades na Política Nacional de Atenção Integral à Saúde do Homem: uma revisão bibliográfica. Saude soc. 22(2). 415-428.

Silveira, C. L.G., Melo, V. F. C., \& Barreto, A. J. R. (2017). Atenção à saúde do homem na atenção primária em saúde. Rev. enferm UFPE online. 11(3). $1528-1535$

Silva, L. E., Freire, F. H. M. A., \& Pereira, R. H. M. (2010). Diferenciais de mortalidade por escolaridade da população adulta brasileira. Cad. Saúde Pública. 32(4). e00019815. 
Research, Society and Development, v. 10, n. 5, e51710515248, 2021

(CC BY 4.0) | ISSN 2525-3409 | DOI: http://dx.doi.org/10.33448/rsd-v10i5.15248

Souza, L.G., \& Siviero, P. C. L. (2015). Diferenciais de mortalidade entre homens e mulheres: Sul de Minas Gerais, 2002 e 2012. Cad. saúde colet. 23(1). 2531. 